A. Shimizu

Nagoya Math. J.

Vol. 71 (1978), 181-198

\title{
CONSTRUCTION OF A SOLUTION OF A CERTAIN EVOLUTION EQUATION II
}

\author{
AKINOBU SHIMIZU
}

Let $D$ be a bounded domain in $R^{d}$ with smooth boundary $\partial D$. We denote by $B_{t}, t \geqq 0$, a one-dimensional Brownian motion. We shall consider the initial-boundary value problem

$$
\begin{gathered}
d u(t, x, \omega)=\left\{a \Delta u+b u\left(1-b_{1} u-b_{2} u^{2}\right)\right\} d t+c u d B_{t}, \\
(t, x) \in[0, \infty) \times D, \\
u(+0, x, \omega)=g(x), \quad x \in D, \\
u(t, \xi, \omega)=0, \quad(t, \xi) \in[0, \infty) \times \partial D,
\end{gathered}
$$

where $a$ and $b$ are positive constants, $b_{1}, b_{2}$ and $c$ are nonnegative constants, and $b_{1}^{2}+b_{2}^{2} \neq 0$. Let $\alpha$ be the positive root of the equation $1-b_{1} \alpha-b_{2} \alpha^{2}=0$. We assume that the initial function $g(x)$ is sufficiently smooth and that $0 \leqq g(x)<\alpha$.

The central aim of this paper is to investigate the asymptotic property of the solution as $t \rightarrow+\infty$, by using the result [7]. In the deterministic case, that is the case $c=0$, the behaviour of the solution is well-known. If the random disturbance $c u d B_{t}$ is involved, the solution presents somewhat complicated behaviour. Therefore, we are interested in the contribution of the coefficient $c$ (Theorem 4.2).

The initial-boundary value problem $(0.1)$ is a stochastic model of population growth with dispersal in theoretical biology (W. H. Fleming [1]). The domain $D$ is the habitat of individuals. The stochastic process $u(t, x, \omega)$ is to be the density of individuals at time $t$ and place $x$ in the habitat $D$. The parameter $\omega$ stands for fluctuation. The term $a \Delta u$, a constant times the Laplace operator, describes the dispersal of individuals. The term $b u\left(1-b_{1} u-b_{2} u^{2}\right)$ means the multiplication rate of individuals. The term $c u d B_{t}$ describes random environmental fluctuations of population growth.

Received September 12, 1977. 
A stochastic model without geographical structure has been discussed in [2]. In the paper, a generalized Volterra system, which is a model of interacting populations of various biological species, is reduced to an ordinary stochastic differential equation. The difference of our model from the model in [2] is only a term $a \Delta u$.

For the deterministic case, it is well-known [11] that

$$
\begin{aligned}
& \lim _{t \rightarrow \infty} u(t, x, \omega)=0, \quad \text { if } \frac{b}{a}<\lambda_{1}, \\
& \lim _{t \rightarrow \infty} u(t, x, \omega)=w(x), \text { a positive stationary solution, if } \frac{b}{a} \geqq \lambda_{1},
\end{aligned}
$$

where $\lambda_{1}$ is the smallest eigenvalue of $-\Delta$ with Dirichlet boundary condition. As soon as the random environmental fluctuations are involved, the condition for survival of individuals largely changes. According to Theorem 4.2 in this paper, we see that

$$
\begin{aligned}
& \lim _{t \rightarrow \infty} u(t, x, \omega)=0 \text { a.e., if } \frac{b}{a}-\frac{1}{2 a} c^{2}<\lambda_{1}, \\
& u(t, x, \omega) \text { never tends to zero a.e., if } \frac{b}{a}-\frac{1}{2 a} c^{2}>\lambda_{1} .
\end{aligned}
$$

Roughly speaking, this result means that the critical size of the habitat $D$ for survival must be larger under the random environmental fluctuations than in the deterministic case.

In $\S 1$, we shall establish a comparison theorem. The existence of a solution will be discussed in $\S 2$. We shall investigate the linear case, $b_{1}=b_{2}=0$, in $\S 3$. In this case, we can get the explicit solution. The algorithm to derive the explicit expression has been obtained by the author [8], using the integral representation of the multiple Wiener integral [4]. Once an explicit expression of the solution is given, it is easy to have a conjecture on the asymptotic behaviour of the solution. Indeed, we can give the affirmative answer to the conjecture in $\S 4$.

The one-dimensional Brownian motion $B_{t}, t \geqq 0$, in the stochastic evolution equation (0.1), which causes the random environmental fluctuations, should now be replaced by a Brownian motion $B_{t}(x, \omega)$ having the parameter $x \in D$, since geographic structure should be taken into account. The author has made an attempt in this scheme in [9], where one is given an explicit form of the solution of a linear stochastic evolu- 
tion equation on a Hilbert space. Although the results in this paper are far from a systematic approach to the theory of infinite dimensional stochastic differential equations, the property obtained in $\S 4$ is well illustrating some evolutionary phenomena arising in population biology.

Before we describe our main results, we list the notations which are used throughout this paper. Let $(\Omega, \widetilde{F}, P$ ) be a probability space, endowed with a right continuous increasing family of $\sigma$-fields $\widetilde{\mho}_{t} . \quad\left\{B_{t}\right\}$ is a one-dimensional $\widetilde{F}_{t}$-Brownian motion. Let $\mathfrak{M}^{\infty}(\mathfrak{F}, D)$ be the family of processes $u(t, x, \omega)$ satisfying the conditions (0.2) and $(0.3)$;

(0.2) $u(t, x, \omega)$ is $(t, x, \omega)$-measurable, and it is $\widetilde{\mho}_{t}$-measurable for each fixed $(t, x) \in[0, T] \times \bar{D}$. $^{1)}$

$$
\sup _{(t, x) \in[0, T] \times \bar{D}} E\left[|u(t, x, \omega)|^{2 k}\right]<+\infty \text { for any } T<+\infty \text { and any positive }
$$
integer $k$.

We denote by $C^{2}(\widetilde{\mho}, D)$ the family of processes $u(t, x, \omega)$ satisfying the conditions $(0.4) \sim(0.7)$;

$$
u \in \mathfrak{M}^{\infty}(\mathfrak{F}, D),
$$

(0.5) $u(t, x, \omega)$ is $(t, x)$-continuous in $[0, \infty) \times \bar{D}$ with probability 1 ,

(0.6) $u(t, x, \omega)$ is twice differentiable in $D$ as a function of $x$, and the derivatives $D_{x} u, D_{x}^{2} u$ are continuous in $(t, x) \in[0, \infty) \times \bar{D}_{.}{ }^{2)}$

$$
\sup _{(t, x) \in[0, T] \times \bar{D}} \sum_{i=0}^{2} E\left[\left|D_{x}^{i} u\right|^{2}\right]<+\infty \quad \text { for each } T<+\infty .
$$

Finally, we will state our main results in $\S 2 \sim \S 4$ precisely. Here, we omit the comparison theorem obtained in $\S 1$, because we need more notations to describe them.

THEOREM 2.5. The initial-boundary value problem (0.1) has a unique nonnegative solution in the class $C^{2}(\widetilde{F}, D)$, if the bounded domain $D$ in d-dimensional Euclidean space $R^{d}$ is taken to be a cuboid, the function $g(x)$ is sufficiently smooth and supp $g(x) \subset D$.

This theorem is a corollary of Theorem 2.4, which gives a more

1) Throughout this paper, we denote by $\bar{D}$ the set $D \cup \partial D$.

2) $D_{x}=\frac{\partial}{\partial x_{i}}, D_{x}^{2}=\frac{\partial^{2}}{\partial x_{i} \partial x_{j}}$ and $D_{x}^{0} u=u$. 
general sufficient condition for the existence and uniqueness of the solution.

THEOREM 3.1. When the coefficients $b_{1}$ and $b_{2}$ are zero, the solution $u(t, x, \omega)$ of the initial-boundary value problem (0.1) can be expressed in the form,

$$
u(t, x, \omega)=\exp \left\{c B_{t}(\omega)-\frac{1}{2} c^{2} t+b t\right\} p(t, x),
$$

where $p(t, x)$ is the solution of the problem,

$$
\begin{aligned}
& \frac{\partial}{\partial t} p(t, x)=a \Delta p(t, x), \quad(t, x) \in[0, \infty) \times D, \\
& p(+0, x)=g(x), \quad x \in D, \\
& p(t, \xi)=0, \quad(t, \xi) \in[0, \infty) \times \partial D .
\end{aligned}
$$

THEOREM 4.2. Let $u(t, x, \omega)$ be the solution of the initial-boundary value problem (0.1). If $-a \lambda_{1}+b-\frac{1}{2} c^{2}<0$, then the equality

$$
P\left[\lim _{t \rightarrow \infty} u(t, x, \omega)=0 \quad \text { for any } x \in D\right]=1 \text {, }
$$

holds. If $-a \lambda_{1}+b-\frac{1}{2} c^{2}>0$, then the equality,

$P[u(t, x, \omega)$ does not converge to zero, as $t \rightarrow+\infty$, for any $x \in D]=1$, holds.

\section{§1. Comparison Theorem}

Let $u(t, x, \omega)$ be a stochastic process belonging to the class $C^{2}(\widetilde{F}, D)$, whose stochastic differential is given by

$$
d u(t, x, \omega)=\left\{a \Delta_{x} u(t, x, \omega)+f(t, x, \omega)\right\} d t+h(u(t, x, \omega)) d B_{t} .
$$

Here, $f(t, x, \omega)$ is $(t, x, \omega)$-measurable, $\widetilde{F}_{t}$-measurable for each fixed $t$ and $x$, and $\int_{0}^{T} E[|f(t, x, \omega)|] d t<+\infty$ for any $x$ and $T<+\infty$. We assume that $v(t, x, \omega) \in C^{2}(\mathscr{\mho}, D)$ be a solution to the stochastic evolution equation,

$$
d v(t, x, \omega)=\left\{a \Delta_{x} v(t, x, \omega)+g(v(t, x, \omega))\right\} d t+h(v(t, x, \omega)) d B_{t} .
$$

We get

THEOREM 1.1. Let $a$ be a nonnegative constant, and $\tau(\omega)$ be a Markov time relative to $\widetilde{\mho}_{t}$. Assume that $h(u)$ is $\frac{1}{2}$-Hölder continuous 
on $R^{1}$, that $g(u)$ can be expressed as the sum of a Lipschitz continuous function and a non-increasing function on $R^{1}$, that the inequality,

$$
f(t, x, \omega) \leqq g(u(t, x, \omega)), \quad(t, x, \omega) \in[0, \tau(\omega)) \times \bar{D} \times \tilde{\Omega},
$$

holds for some $\omega$-set $\tilde{\Omega}$ satisfying $P(\widetilde{\Omega})=1$, and that the conditions

$$
P[u(0, x, \omega) \leqq v(0, x, \omega) \quad \text { for any } x \in \bar{D}]=1
$$

and

(1.5) $P[u(t, \xi, \omega) \leqq v(t, \xi, \omega) \quad$ for any $\xi \in \partial D$ and any $t \in[0, \tau(\omega))]=1$

hold. Then, the equality

(1.6) $P[u(t, x, \omega) \leqq v(t, x, \omega) \quad$ for any $x \in \bar{D}$ and any $t \in[0, \tau(\omega))]=1$

holds.

Proof. Let us define a sequence of functions $\varphi_{n}(u)$ as follows;

$$
\begin{aligned}
& \varphi_{n}(u) \text { are of } C^{2} \text {-class, } \varphi_{n}(u) \geqq 0, \varphi_{n}(u) \uparrow \max (0, u) \\
& 0 \leqq \varphi_{n}^{\prime}(u) \leqq 1, \quad \varphi_{n}^{\prime}(u) \rightarrow 1(u>0) \\
& u \varphi_{n}^{\prime \prime}(u) \text { are uniformly bounded, and } \varphi_{n}^{\prime \prime}(u) \rightarrow 0(n \rightarrow+\infty)
\end{aligned}
$$

We put $\tau_{r}(\omega)=\inf \left\{t ; \sup _{x \in \bar{D}} \max (|u(t, x, \omega)|,|v(t, x, \omega)|)>r\right\}$, and $t^{\prime}=t$ $\wedge \tau(\omega) \wedge \tau_{r}(\omega)$. By using Itô's formula, we get

$$
\begin{aligned}
& e^{-k t^{\prime}} \varphi_{n}\left(u\left(t^{\prime}, x, \omega\right)-v\left(t^{\prime}, x, \omega\right)\right) \\
&=- k \int_{0}^{t^{\prime}} e^{-k s} \varphi_{n}(u-v) d s \\
&+\int_{0}^{t^{\prime}} e^{-k s} \varphi_{n}^{\prime}(u-v)\{a \Delta(u-v)+f-g(v)\} d s \\
&+\int_{0}^{t^{\prime}} e^{-k s} \varphi_{n}^{\prime}(u-v)\{h(u)-h(v)\} d B_{s} \\
&+\int_{0}^{t^{\prime}} \frac{1}{2} e^{-k s} \varphi_{n}^{\prime \prime}(u-v)\{h(u)-h(v)\}^{2} d s .
\end{aligned}
$$

Hence, we have

$$
\begin{aligned}
& E\left[e^{-k t^{\prime}} \varphi_{n}\left(u\left(t^{\prime}, x, \omega\right)-v\left(t^{\prime}, x, \omega\right)\right)\right] \\
& \quad \leqq-k E\left[\int_{0}^{t^{\prime}} e^{-k s} \varphi_{n}(u-v) d s\right] \\
& \quad+E\left[\int_{0}^{t^{\prime}} e^{-k s} \varphi_{n}^{\prime}(u-v)\{a \Delta(u-v)+f(s, x, \omega)-g(u(s, x, \omega))\right.
\end{aligned}
$$




$$
\begin{gathered}
+g(u)-g(v)\} d s] \\
+k^{\prime} E\left[\int_{0}^{t^{\prime}} e^{-k s} \varphi_{n}^{\prime \prime}(u-v)|u-v| d s\right] \\
\leqq-k E\left[\int_{0}^{t^{\prime}} e^{-k s} \varphi_{n}(u-v) d s\right] \\
+E\left[\int_{0}^{t^{\prime}} e^{-k s} \varphi_{n}^{\prime}(u-v)\left\{a \Delta(u-v)+k^{\prime \prime}|u-v|\right\} d s\right] \\
+k^{\prime} E\left[\int_{0}^{t^{\prime}} e^{-k s} \varphi_{n}^{\prime \prime}(u-v)|u-v| d s\right],
\end{gathered}
$$

because of the inequality (1.3) and $g(u)-g(v) \leqq k^{\prime \prime}|u-v|,(u>v)$. Letting $n$ tend to $+\infty$, we have

$$
\begin{aligned}
& E\left[e^{-k t^{\prime}} \max (0, u-v)\right] \\
& \leqq \\
& \quad\left(k^{\prime \prime}-k\right) E\left[\int_{0}^{t^{\prime}} e^{-k s} \max (0, u-v) d s\right] \\
& \quad+E\left[\int_{0}^{t^{\prime}} e^{-k s} \chi_{(u>v)} a \Delta(u-v) d s\right] .
\end{aligned}
$$

Let the constant $k$ be sufficiently large, then we have

$$
\begin{aligned}
& E\left[e^{-k t^{\prime}} \max (0, u-v)\right] \\
& \quad \leqq E\left[\int_{0}^{t^{\prime}} e^{-k s} \chi_{(u>v)} a \Delta(u-v) d s\right] .
\end{aligned}
$$

Hence, we have

$$
\begin{aligned}
\int_{D} E\left[e^{-k t^{\prime}}\right. & \left.\max \left(0, u\left(t^{\prime}, x, \omega\right)-v\left(t^{\prime}, x, \omega\right)\right)\right] d x \\
& \leqq \int_{D} E\left[\int_{0}^{t^{\prime}} e^{-k s} \chi_{(u>v)} a \Delta(u-v) d s\right] d x \\
& =E\left[\int_{0}^{t^{\prime}} e^{-k s}\left(\int_{D} \chi_{(u>v)} a \Delta(u-v) d x\right) d s\right] .
\end{aligned}
$$

By the next Lemma 1.2, we must have

$$
\int_{D} \chi_{(u>v)} a \Delta(u-v) d x \leqq 0
$$

therefore, we obtain

$$
\int_{D} E\left[e^{-k t^{\prime}} \max \left(0, u\left(t \wedge \tau \wedge \tau_{r}, x, \omega\right)-v\left(t \wedge \tau \wedge \tau_{r}, x, \omega\right)\right)\right] d x \leqq 0 .
$$

Noting the fact that $\tau_{r}(\omega) \rightarrow+\infty, r \rightarrow+\infty$, we get the conclusion of 
this theorem.

To complete the proof of Theorem 1.1, we will prove

LEMMA 1.2. Assume that a function $\psi(x)$ belongs to $c^{1}(\bar{D}) \cap C^{2}(D),{ }^{3)}$ that $\int_{D}\left|\frac{\partial^{2}}{\partial x_{i}^{2}} \psi(x)\right| d x<+\infty(i=1,2, \cdots, d)$, and that $\psi(\xi) \leqq 0$ for each $\xi \in \partial D$. Then, the inequality

$$
\int_{D \cap\{x ; \psi(x)>0\}} \Delta \psi(x) d x \leqq 0
$$

holds.

Proof. We will prove the inequality under the assumption $\psi(x)$ $\in C^{2}(\bar{D})$. It is sufficient to show that $\int_{D \cap\{\psi(x)>0\}} \frac{\partial^{2}}{\partial x_{i}^{2}} \psi(x) d x \leqq 0$ for each $i$. For fixed $x_{1}, x_{2}, \cdots, x_{i-1}, x_{i+1}, \cdots, x_{d}$, we put

$$
L_{i}=\left\{x=\left(x_{1}, \cdots, x_{d}\right) ; x \in D \cap\{\psi(x)>0\}\right\} .
$$

Clearly, it is enough to see that $\int_{L_{i}} \frac{\partial^{2}}{\partial x_{i}^{2}} \psi(x) d x_{i} \leqq 0$. Since the set $L_{i}$ is expressed as the sum of countably many open intervals, the assertion of Lemma 1.2 is reduced to the following statement;

$$
\int_{\alpha}^{\beta} \frac{\partial^{2}}{\partial x_{i}^{2}} \psi(x) d x_{i} \leqq 0, \text { under the conditions } \psi(\alpha)=\psi(\beta)=0 \text {, and } \psi(x)>0,
$$

$x_{i} \in(\alpha, \beta)$. This is obvious. We omit the proof under the assumptions of this Lemma, because it is easy to get it modifying the above proof.

When the constant $a$ equals zero, Theorem 1.1 gives a comparison theorem for ordinary stochastic differential equations [10]. On the other hand, putting $c=0$, Theorem 1.1 turns out a comparison theorem for partial differential equations of parabolic type, which is well-known.

Next, we will give some results, which can be immediately deduced from Theorem 1.1. We shall use them in $\S 2$ and $\S 4$.

COROLlary 1.3. (i) Let a be a nonnegative constant, and $\tau(\omega)$ be a Markov time relative to $\mathfrak{F}_{t}$. Assume that $h(u)$ is $\frac{1}{2}$-Hölder continuous on $[0, \infty)$, that $g(u)$ can be expressed as the sum of a Lipschitz conin $D$.

3) $\psi(x)$ is continuously differentiable in $\bar{D}$, and twice continuously differentiable 
tinuous function and a nonincreasing function on $[0, \infty)$, that the processes $u(t, x, \omega)$ and $v(t, x, \omega)$ are nonnegative, and that the conditions (1.3) (1.4) and (1.5) are satisfied. Then, (1.6) holds.

(ii) Let $a$ be a nonnegative constant. In addition to the assumptions on the functions $h(u)$ and $g(u)$ in the above (i), we assume that $h(0)$ $=g(0)=0$. Let a process $v(t, x, \omega),(t, x) \in[0, \infty) \times \bar{D}$, be a solution of the stochastic evolution equation (1.2) satisfying the conditions;

$$
\begin{array}{ll}
P[v(0, x, \omega) \geqq 0 & \text { for any } x \in \bar{D}]=1, \\
P[v(t, \xi, \omega) \geqq 0 & \text { for any }(t, \xi) \in[0, \infty) \times \partial D]=1 .
\end{array}
$$

Then, the equality

$$
P[v(t, x, \omega) \geqq 0 \quad \text { for any }(t, x) \in[0, \infty) \times \bar{D}]=1
$$

holds.

COROLlaRY 1.4. (i) In the assumptions in Corollary 1.3 (i), we replace the conditions (1.3)(1.4) and (1.5) by the following conditions;

$$
f(t, x, \omega)=g(u(t, x, \omega)), \quad(t, x, \omega) \in[0, \tau(\omega)) \times \bar{D} \times \tilde{\Omega},
$$

holds for some $\omega$-set $\tilde{\Omega}$ satisfying $P(\tilde{\Omega})=1$,

$$
P[u(0, x, \omega)=v(0, x, \omega) \quad \text { for any } x \in \bar{D}]=1,
$$

$$
P[u(t, \xi, \omega)=v(t, \xi, \omega) \quad \text { for any }(t, \xi) \in[0, \tau(\omega)) \times \partial D]=1 .
$$

Then, the equality

$$
P[u(t, x, \omega)=v(t, x, \omega) \quad \text { for any }(t, x) \in[0, \tau(\omega)) \times \bar{D}]=1
$$

holds.

(ii) Under the same assumptions on the functions $h(u), g(u)$ and the constant a as in Corollary 1.3 (ii), the uniqueness of nonnegative solutions of the stochastic evolution equation (1.2), which satisfy the initial condition

$$
P[v(0, x, \omega)=g(x) \geqq 0 \quad \text { for any } x \in \bar{D}]=1
$$

and the boundary condition

$$
P[v(t, \xi, \omega)=0 \quad \text { for any }(t, \xi) \in[0, \infty) \times \partial D]=1,
$$

holds. 


\section{§2. Existence of a solution}

The aim of this section is to establish the existence of a solution of the initial-boundary value problem (0.1). We can prove the existence, when the domain is a cuboid. Let $p(t, x, y)$ be the fundamental solution of the heat equation,

$$
\frac{\partial}{\partial t} u=a \Delta u
$$

with boundary condition,

$$
u(t, \xi)=0, \quad \xi \in \partial D .
$$

First, we consider the following equation of integral type.

$$
\begin{aligned}
u(t, x, \omega)= & \int_{D} p(t, x, y) g(y) d y \\
& +\int_{0}^{t} d s \int_{D} p(t-s, x, y) f(u(s, \dot{y}, \omega)) d y \\
& +\int_{0}^{t}\left\{\int_{D} p(t-s, x, y) h(u(s, y, \omega)) d y\right\} d B_{s}
\end{aligned}
$$

where the function $f(u)$ and the derivative $h^{\prime}(u)$ are Lipschitz continuous. The existence of a solution of (2.3), which belongs to the class $\mathfrak{M}^{\infty}(\mathfrak{\mho}, D)$, is proved by the successive approximation. We expect that the solution of (2.3) satisfies (0.1). If the domain $D$ coincides with $R^{d}$, we can prove this fact by the same argument as in B. L. Rozovskii [7]. But, in this paper, we consider the initial-boundary value problem (0.1) with bounded domain $D$, so we can not apply Rozovskii's method.

We assume that the bounded domain $D$ is given by

$$
D=\left\{\left(x_{1}, x_{2}, \cdots, x_{d}\right) \in R^{d} ; 0<x_{k}<L_{k}, k=1,2, \cdots, d\right\} .
$$

Then, the fundamental solution $p(t, x, y)$ of (2.1) with boundary condition (2.2) is represented in the form,

$$
\begin{aligned}
p(t, x, y)=\frac{1}{(2 \sqrt{\pi a t})^{d}} \prod_{k=1}^{d} \sum_{n=-\infty}^{+\infty} & {\left[\exp \left\{-\frac{\left(x_{k}-y_{k}+2 n L_{k}\right)^{2}}{4 a t}\right\}\right.} \\
& \left.-\exp \left\{-\frac{\left(x_{k}+y_{k}+2 n L_{k}\right)^{2}}{4 a t}\right\}\right] .
\end{aligned}
$$

For a stochastic process $u(t, x, \omega), x \in D$, we define an extension $v(t, x, \omega)$, $x \in R^{d}$, of the process $u(t, x, \omega)$ such that the process $v(t, x, \omega)$ satisfies 
the conditions $(2.6) \sim(2.8)$ with probability 1 for any $(t, x) \in[0, \infty) \times D$;

$$
\begin{gathered}
\qquad(t, x, \omega)=u(t, x, \omega), \\
v\left(t, x_{1}+2 n_{1} L_{1}, x_{2}+2 n_{2} L_{2}, \cdots, x_{d}+2 n_{d} L_{d}, \omega\right) \\
=v\left(t, x_{1}, x_{2}, \cdots, x_{d}, \omega\right) \quad \text { for any integer } n_{1}, n_{2}, \cdots, n_{d}, \\
v\left(t, \varepsilon_{1} x_{1}, \varepsilon_{2} x_{2}, \cdots, \varepsilon_{d} x_{d}, \omega\right)=\varepsilon_{1} \varepsilon_{2} \cdots \varepsilon_{d} v\left(t, x_{1}, x_{2}, \cdots, x_{d}, \omega\right)
\end{gathered}
$$

for any $\varepsilon_{k}= \pm 1$. Furthermore, we define

$$
\tilde{f}(u)= \begin{cases}f(u), & u \geqq 0 \\ -f(-u), & u<0 .\end{cases}
$$

Then, we have

LEMMA 2.1. If $u(t, x, \omega) \geqq 0$ almost everywhere for any $(t, x)$ $\in[0, \infty) \times D$, then the equality

$$
\begin{aligned}
& \int_{D} p(-s, x, y) f(u(s, y, \omega)) d y \\
&=\int_{R^{d}} \frac{1}{(2 \sqrt{\pi a(t-s)})^{d}} \exp \left\{-\frac{(x-y)^{2}}{4 a(t-s)}\right\} \tilde{f}(v(s, y, \omega)) d y,
\end{aligned}
$$

holds, where the process $v$ is the extension of $u$ defined by (2.6) (2.8). Conversely, if a process $v(t, x, \omega), x \in R^{d}$, satisfies (2.7), (2.8) and $v(t, x, \omega)$ $\geqq 0$ a.e. for $(t, x) \in[0, \infty) \times D$, then the process $u(t, x, \omega)$ defined by $(2.6)$ satisfies (2.10).

We will omit the proof of Lemma 2.1, because it is not difficult.

Making use of Lemma 2.1, we see that the integral equation (2.3) can be reduced to the equation,

$$
\begin{aligned}
v(t, x, \omega)= & \int_{R^{d}} p_{0}(t, x, y) \tilde{g}(y) d y \\
& +\int_{0}^{t} d s \int_{R^{d}} p_{0}(t-s, x, y) \tilde{f}(v(s, y, \omega)) d y \\
& +\int_{0}^{t} \int_{R^{d}} p_{0}(t-s, x, y) \tilde{h}(v(s, y, \omega)) d y d B_{s},
\end{aligned}
$$

where $p_{0}(t, x, y)=\frac{1}{(2 \sqrt{\pi a t})^{d}} \exp \left\{-\frac{(x-y)^{2}}{4 a t}\right\}$, the function $\tilde{g}(x), x \in R^{d}$, is an extention of $g(x), x \in D$, such that $\tilde{g}(x)$ has the properties corresponding to (2.7), (2.8), and the function $\tilde{h}(u)$ is defined by (2.9). 
Since we can apply the Rozovskii's method to the integral equation (2.11), we get the following lemma.

LEMMA 2.2. (i) Assume that the functions $f(u)$ and $h(u)$ are Lipschitz continuous, that $f(0)=h(0)=0$, and that the function $g(x)$ is bounded measurable. Then, the integral equation (2.11) has a unique solution in the class $\mathfrak{M}^{\infty}\left(\mathfrak{\mho}, R^{d}\right)$.

(ii) In addition to the assumptions in (i), we assume that $f(u)$ and the derivative $h^{\prime}(u)$ is Lipschitz continuous, and that $g(x)$ is a sufficiently smooth function with supp $g(x) \subset D$. Then, the solution of (2.11) belongs to the class $C^{2}\left(\widetilde{\mho}, R^{d}\right)$ and it satisfies the stochastic evolution equation,

$$
d v(t, x, \omega)=\{a \Delta v+\tilde{f}(v)\} d t+\tilde{h}(v) d B_{t} .
$$

(iii) In addition to the assumptions in (ii), we assume that the function $g(x), x \in D$, is nonnegative. Then, the solution $v(t, x, \omega)$ of (2.12) is nonnegative in $[0, \infty) \times D$ almost surely, and it satisfies $(2.7)(2.8)$.

Proof. (i ) Since the functions $\tilde{f}(u)$ and $\tilde{h}(u)$ are Lipschitz continuous under our assumptions, we can apply the successive approximation. Let us define an operator $S$ as follows:

$$
\begin{aligned}
S u= & \int_{R^{d}} p_{0}(t, x, y) \tilde{g}(y) d y \\
& +\int_{0}^{t} d s \int_{R^{d}} p_{0}(t-s, x, y) \tilde{f}(u(s, y, \omega)) d y \\
& +\int_{0}^{t} \int_{R^{d}} p_{0}(t-s, x, y) \tilde{h}(u(s, y, \omega)) d y d B_{s} .
\end{aligned}
$$

We put $u_{1}=\int_{R^{d}} p_{0}(t, x, y) \tilde{g}(y) d y$. We can easily verify that $S^{n} u_{1}$ converges in norm $\left.\|u\|_{N}=\sup _{(t, x) \in[0, \tau] \times R^{d}} E\left[|u(t, x, \omega)|^{2 N}\right]\right)^{1 / 2 N}$, where $N$ is an arbitrary integer. Hence, we get the conclusion.

(ii) Since the support of the function $g(x)$ is contained in $D$, the extended function $\tilde{g}(x)$ is sufficiently smooth in $R^{d}$. Applying Rozovskii's method [7], we get the conclusion.

(iii) If a function $w(x)$ satisfies the conditions (2.7)(2.8), it is easy to see that the function $\int_{R^{d}} p_{0}(t, x, y) w(y) d y$ also satisfies $(2.7)(2.8)$. Noting the above fact, we see that $u_{1}$ satisfies (2.7)(2.8), and that $S^{n} u_{1}$ satisfies 
(2.7)(2.8). Since the sequence $S^{n} u_{1}$ converges to the solution in norm $\|\cdot\|_{N}$, so the solution satisfies $(2.7)(2.8)$. The fact that $v(t, x, \omega) \geqq 0$ a.e. for $(t, x) \in[0, \infty) \times D$ follows from Corollary 1.3 (ii).

By Lemma 2.1 and Lemma 2.2, we get

Proposition 2.3. Assume that the domain $D$ is given by (2.4), that $f(u)$ and the derivative $h^{\prime}(u)$ are Lipschitz continuous, that $f(0)=h(0)$ $=0$, that the function $g(x)$ is nonnegative and sufficiently smooth, and that the support of $g(x)$ is contained in $D$. Then, the initial-boundary value problem

$$
\begin{aligned}
& d u(t, x, \omega)=\{a \Delta u+f(u)\} d t+h(u) d B_{t}, \\
& u(0, x, \omega)=g(x), \quad x \in D, \\
& u(t, \xi, \omega)=0, \quad \xi \in \partial D,
\end{aligned}
$$

has a unique nonnegative solution in $C^{2}(\mathscr{\mho}, D)$.

Next, we should relax the condition on the function $f(u)$ in Proposition 2.3, because the function $b u\left(1-b_{1} u-b_{2} u^{2}\right)$ which appears in $(0,1)$ is not Lipschitz continuous.

Theorem 2.4. Assume that the domain $D$ is given by (2.4), that the function $f(u)$ is written in the form,

$$
f(u)=f_{1}(u)-f_{2}(u),
$$

with a Lipschitz continuous function $f_{1}(u)$ and a nonnegative increasing locally Lipschitz function $f_{2}(u)$, that the derivative $h^{\prime}(u)$ is Lipschitz continuous, that $f_{1}(0)=f_{2}(0)=h(0)=0$, and that the function $g(x)$ is nonnegative and sufficiently smooth, and that supp $g(x) \subset D$. Then, the initial-boundary value problem (2.13) has a unique nonnegative solution in the class $C^{2}(\mathfrak{F}, D)$.

Proof. We put

$$
f_{2}^{(n)}(u)= \begin{cases}f_{2}(u), & \text { if } f_{2}(u)<n+1 \\ n+1 & \text { if } f_{2}(u) \geq n+1\end{cases}
$$

and

$$
f^{(n)}(u)=f_{1}(u)-f_{2}^{(n)}(u) .
$$

We consider the stochastic evolution equation, 


$$
d u=\left\{a \Delta u+f^{(n)}(u)\right\} d t+h(u) d B_{t},
$$

with initial condition,

$$
u(+0, x, \omega)=g(x), \quad x \in D,
$$

and the boundary condition,

$$
u(t, \xi, \omega)=0, \quad \xi \in \partial D .
$$

By Proposition 2.3, we see that (2.14) has a unique solution $u^{(n)}(t, x, \omega)$. We define a Markov time $\tau_{n}(\omega)$ by

$$
\tau_{n}(\omega)=\inf \left\{t ; f_{2}\left(u^{(n)}(t, x, \omega)\right)>n \quad \text { for some } x \in D\right\} .
$$

From Corollaries 1.3 and 1.4, it follows that $u^{(n)}(t, x, \omega) \geqq u^{(n+1)}(t, x, \omega)$, that $\tau_{n}(\omega) \leqq \tau_{n+1}(\omega)$, and that $u^{(n)}(t, x, \omega)=u^{(n+1)}(t, x, \omega)$ if $t<\tau_{n}(\omega)$. We put

$$
u(t, x, \omega)=u^{(n)}(t, x, \omega), \quad \text { if } t<\tau_{n}(\omega),
$$

and

$$
\tau_{\infty}(\omega)=\lim _{n \rightarrow \infty} \tau_{n}(\omega)
$$

The stochastic process $u(t, x, \omega)$ defined by (2.15) satisfies (2.13) when $t<\tau_{\infty}(\omega)$, so that it is enough to verify $\tau_{\infty}(\omega)=+\infty$ to complete the proof of the existence. Let $w(t, x, w)$ be the solution of the stochastic evolution equation,

$$
\begin{aligned}
& d w(t, x, \omega)=\left\{a \Delta w+f_{1}(w)\right\} d t+h(w) d B_{t}, \\
& w(+0, x, \omega)=g(x), \quad x \in D, \\
& w(t, \xi, \omega)=0, \quad \xi \in \partial D .
\end{aligned}
$$

Then, by Theorem 1.1 , we get that $w(t, x, \omega) \geqq u^{(n)}(t, x, \omega)$. Hence, $f_{2}(w(t, x, \omega)) \geqq f_{2}\left(u^{(n)}(t, x, \omega)\right)$. Therefore, we have

$$
\begin{aligned}
\tau_{n}(\omega) & \geqq \inf \left\{t ; f_{2}(w(t, x, \omega))>n \quad \text { for some } x \in D\right\} \\
& \equiv \hat{\tau}_{n}(\omega) .
\end{aligned}
$$

It is easy to see that $\hat{\tau}_{n}(\omega) \rightarrow+\infty$, because $w(t, x, \omega)$ is $(t, x)$-continuous in $[0,+\infty) \times D$ with probability 1 . Hence, we get $\tau_{\infty}(\omega)=+\infty$. The uniqueness of solutions follows from Corollary 1.4 (ii). The proof is complete.

The next theorem is only a corollary of Theorem 2.4. But, it is 
sufficient for the central aim of this paper.

THEOREM 2.5. The initial-boundary value problem (0.1) has a unique nonnegative solution in the class $C^{2}(\mathfrak{F}, D)$, if the bounded domain $D$ in $d$-dimensional Euclidean space $R^{d}$ is a cuboid, the function $g(x)$ is sufficiently smooth, nonnegative and supp $g(x) \subset D$.

\section{§3. Linear stochastic evolution equation}

In this section, we consider the initial-boundary value problem of the linear stochastic evolution equation;

$$
d u=\{a \Delta u+b u\} d t+c u d B_{t},
$$

$$
u(t, \xi, \omega)=0, \quad \xi \in \partial D,
$$

where $D$ is a bounded domain in $R^{d}$ with smooth boundary $\partial D$. The initial-boundary value problem $(0.1)$ is reduced to the problem (3.1) $\sim$ (3.3), if the constants $b_{1}$ and $b_{2}$ in (0.1) equals zero.

THEOREM 3.1. When the constants $b_{1}$ and $b_{2}$ are zero, the solution $u(t, x, \omega)$ of the initial-boundary value problem (0.1) can be expressed in the form,

$$
u(t, x, \omega)=\exp \left\{c B_{t}(\omega)-\frac{1}{2} c^{2} t+b t\right\} p(t, x),
$$

where $p(t, x)$ is the solution of the problem,

$$
\begin{aligned}
& \frac{\partial}{\partial t} p=a \Delta p(t, x), \quad(t, x) \in[0, \infty) \times D, \\
& p(+0, x)=g(x), \quad x \in D, \\
& p(t, \xi)=0, \quad(t, \xi) \in[0, \infty) \times \partial D .
\end{aligned}
$$

Proof. The integral equation corresponding to (3.1) can be written as follows;

$$
\begin{aligned}
u(t, x, \omega)= & \int_{D} p(t, x, y) g(y) d y+\int_{0}^{t}\left\{\int_{D} p(t-s, x, y) b u(s, y, \omega) d y\right\} d s \\
& +\int_{0}^{t}\left\{\int_{D} p(t-s, x, y) c u(s, y, \omega) d y\right\} d B_{s} .
\end{aligned}
$$

By the same argument as the proof of Theorem 1 in [8], we obtain the 
formula (3.4). We can easily verify by Itô's formula that the process $u(t, x, \omega)$ given by (3.4) satisfies (3.1). Obviously, the process $u(t, x, \omega)$ satisfies (3.2) and (3.3).

Let $\left\{\lambda_{n}\right\}$ be the eigenvalues of $(\lambda+\Delta) u=0$ with boundary condition $\left.u\right|_{\partial D}=0$, such that $\lambda_{1}<\lambda_{2} \leqq \lambda_{3} \leqq \cdots$, and $\left\{\varphi_{n}\right\}$ be the eigenfunctions corresponding to $\left\{\lambda_{n}\right\}$. Then, the function $p(t, x)$ in Theorem 3.1 can be expressed in the form,

$$
p(t, x)=\sum_{n=1}^{\infty} e^{-a \lambda_{n} t} A_{n} \varphi_{n}(x) .
$$

The right-hand side of (3.5) converges uniformly in $(t, x) \in[\delta, \infty) \times \bar{D}$ for each $\delta>0$. The conditions $g(x)$ and $g(x) \not \equiv 0$ imply $A_{1} \varphi_{1}(x)>0, x \in D$.

CoRollary 3.2. The inequality $-a \lambda_{1}+b-\frac{1}{2} c^{2}<0$ implies

$$
P\left[\lim _{t \rightarrow \infty} u(t, x, \omega)=0 \quad \text { for any } x \in D\right]=1,
$$

and $-a \lambda_{1}+b-\frac{1}{2} c^{2}>0$ implies

$$
P\left[\lim _{t \rightarrow \infty} u(t, x, \omega)=+\infty \quad \text { for any } x \in D\right]=1 .
$$

Proof. Combining the fact, $P\left[\lim _{t \rightarrow \infty} \frac{B_{t}}{t}=0\right]=1$, with Theorem 3.1, we get the conclusion of Corollary 3.2.

\section{§4. Asymptotic behaviour of the solution}

Let $u(t, x, \omega)$ be the solution of the initial-boundary value problem (0.1). Our concern of this section is whether $u(t, x, \omega)$ converges to zero or not as $t \rightarrow+\infty$.

First, we assume that $-a \lambda_{1}+b-\frac{1}{2} c^{2}<0$. By Corollary 1.3, the solution $u(t, x, \omega)$ of $(0.1)$ is not greater than the solution of $(3.1) \sim(3.3)$. Combining this with Corollary 3.2, we get

$$
P\left[\lim _{t \rightarrow \infty} u(t, x, \omega)=0 \quad \text { for any } x \in D\right]=1
$$

Next, we assume that $-a \lambda_{1}+b-\frac{1}{2} c^{2}>0$. We can choose real numbers $\alpha, \beta$ such that $b=\alpha+\beta, \alpha>a \lambda_{1}$, and $\beta>\frac{1}{2} c^{2}$. Let us define a function $\hat{u}(t, x)$ and a process $X_{t}(\omega)$ by (4.1), (4.2); 


$$
\begin{aligned}
& \frac{\partial \hat{u}}{\partial t}=a \Delta \hat{u}(t, x)+\alpha \hat{u}(t, x)\left(1-\frac{1}{\hat{k}} \hat{u}(t, x)\right), \\
& \hat{u}(+0, x)=\hat{g}(x), \quad 0 \leqq \hat{g}(x)<\min (g(x), \hat{k}), \quad \hat{g}(x) \neq 0, \\
& \hat{u}(t, \xi)=0, \quad \text { if } \xi \in \partial D, \\
& \quad d X_{t}=\beta X_{t}\left(1-\gamma_{1} X_{t}-\gamma_{2} X_{t}^{2}\right) d t+c X_{t} d B_{t}, \\
& X_{0} \equiv 1,
\end{aligned}
$$

where $\hat{k}$ is a positive constant, and $\gamma_{1}, \gamma_{2}$ are nonnegative constants. We shall determine $\hat{k}, \gamma_{1}$ and $\gamma_{2}$ in the next lemma.

LEMMA 4.1. There exist $\hat{k}>0, \gamma_{1}, \gamma_{2} \geqq 0$ such that the equality, $P\left[u(t, x, \omega) \geqq \hat{u}(t, x) X_{t}(\omega)\right.$, for any $x \in D$, any $\left.t \geqq 0\right]=1$, holds.

Proof. We put $w(t, x, \omega)=\hat{u}(t, x) X_{t}(\omega)$. Let us calculate the stochastic differential of $w(t, x, \omega)$. Then, we have

$$
\begin{aligned}
d w= & \{a \Delta w+b w\} d t+c w d B_{t} \\
& -w\left\{\frac{\alpha}{\hat{k}} \hat{u}+\beta \gamma_{1} \frac{w}{\hat{u}}+\beta \gamma_{2} \frac{w^{2}}{\hat{u}^{2}}\right\} d t .
\end{aligned}
$$

Since $\hat{g} \leqq \hat{k}$, so we have $0 \leqq \hat{u} \leqq \hat{k}$ by the comparison theorem on partial differential equations, which is included in Theorem 1.1. Therefore, we have

$$
-w\left\{\frac{\alpha}{\hat{k}} \hat{u}+\beta \gamma_{1} \frac{w}{\hat{u}}+\beta \gamma_{2} \frac{w^{2}}{\hat{u}^{2}}\right\} \leqq-\frac{\beta \gamma_{1}}{\hat{k}} w^{2}-\frac{\beta \gamma_{2}}{\hat{k}^{2}} w^{3} \cdot{ }^{4)}
$$

Let $k$ be any positive number, and let $\gamma_{1}$ and $\gamma_{2}$ be the constants such that

$$
\frac{\beta \gamma_{1}}{\hat{k}}=b b_{1}, \quad \frac{\beta \gamma_{2}}{\hat{k}^{2}}=b b_{2} .
$$

Then, it follows from Corollary 1.3 that the equality,

$$
P[u(t, x, \omega) \geqq w(t, x, \omega) \quad \text { for any } x \in \bar{D} \text { and } t \geqq 0]=1,
$$

holds.

Since $\alpha>\gamma_{1}$, we have

$$
u(t, x) \rightarrow S(x), \text { and } 0<S(x) \leqq k, x \in D[11]
$$

4) It is enough to consider only the case $\hat{u} \neq 0$. 
On the other hand, it is known in the theory of one-dimensional diffusion processes that the path of $X_{t}(\omega)$ does not converge to zero with probability 1 under the condition $\beta>\frac{1}{2} c^{2}$. Combining these facts with Lemma 4.1, we obtain

THEOREM 4.2. Let $u(t, x, \omega)$ be the solution of the initial-boundary value problem (0.1). If $-a \lambda_{1}+b-\frac{1}{2} c^{2}<0$, then the equality

$$
P\left[\lim _{t \rightarrow \infty} u(t, x, \omega)=0 \quad \text { for any } x \in \bar{D}\right]=1
$$

holds. If $-a \lambda_{1}+b-\frac{1}{2} c^{2}>0$, then the equality

$$
\begin{array}{r}
P[u(t, x, \omega) \text { does not converge to zero, as } t \rightarrow+\infty, \\
\text { for any } x \in D]=1
\end{array}
$$

holds.

Acknowledgement: The author thanks the referee for his suggestion on way of expressing the results of Theorem 1.1, Corollaries 1.3, and 1.4.

\section{Addendum}

After this paper was submitted for publication, the author presented a note 'Asymptotic property of the solution of a certain stochastic evolution equation' to 'Proceedings of the International Symposium on Mathematical Topics in Biology, Kyoto, Japan 1978', where he discussed a stochastic evolution equation similar to Eq. (0.1) in this paper. The evolution equation, which he discussed in the above note, seems more natural as a stochastic population growth model in population biology.

\section{REFERENCES}

[1] W. H. Fleming, Distributed parameter stochastic systems in population biology, m.s., Brown University, Providence, R. I.

[2] N. S. Goel, S. C. Maitra and E. W. Montroll, On the Volterra and other nonlinear models of interacting populations, Rev. Modern Phys. 43 (2) (1971).

[ 3 ] M. Golubitsky and V. Guillemin, Stable mappings and their singularities, SpringerVerlag, 1973.

[ 4 ] T. Hida, Analysis of Brownian functionals, Carleton University lecture notes, 1975.

[5] Y. Okabe and A. Shimizu, On the pathwise uniqueness of solutions of stochastic differential equations, J. Math. Kyoto Univ. 15 (2) (1975).

[6] A. Okubo, Biology and diffusion (in Japanese), Tsukiji-shokan, 1975.

[ 7 ] B. L. Rozovskii, On stochastic differential equations with partial derivatives (in Russian), Math. Sb. 96(138), 2 (1975).

[8] A. Shimizu, Construction of a solution of a certain evolution equation, Nagoya 
Math. J. 66 (1977).

[9] - Construction of a solution of linear stochastic evolution equations on a Hilbert space, to appear.

[10] T. Yamada, On a comparison theorem for solutions of stochastic differential equations and its applications, J. Math. Kyoto Univ. 13 (1973).

[11] M. Yamaguti, Mathematics in non-linear phenomena (in Japanese) Asakurashoten, 1972.

Nagoya Institute of Technology 